\title{
Patchouli (Pogostemon cablin Benth.): botany, agrotechnology and biotechnological aspects
}

\begin{abstract}
Patchouli (Pogostemon cablin Benth.) is an industrially valued aromatic medicinal plant currently having a huge demand for its essential oil. It is widely used in flavor and fragrance industries as well as in pharmaceuticals. Clonal propagation is therefore obligatory to obtain homogeneous patchouli plants from preferred germplasm in order to meet the global need. At present, farmers are showing keen interest towards its large scale cultivation mainly in the tropical and sub-tropical countries. Understanding the plant biology, chemistry and other uses allows better utilization of any industrial crops. Therefore, the present review discusses on the botany, agronomy and biotechnological aspects of patchouli. Also, the current market demand for patchouli raw material is being reviewed by highlighting the possible exploration of modern techniques in identifying the adulterants to authenticate this herb. This review provides a comprehensive information on both basic and applied aspects of patchouli crop cultivation and management. The applied aspect of patchouli biotechnology allows one to explore the newer potential for various industrial uses and improve the rural economy.
\end{abstract}

Keyword: Patchouli; Herbal medicine; Essential oil; Perfume; Aromatherapy; Adulteration; Industrial crop 